\title{
PERSISTENT SCIATIC ARTERY ANEURYSM: A RARE EMBRYOLOGICAL VASCULAR VARIANT
}

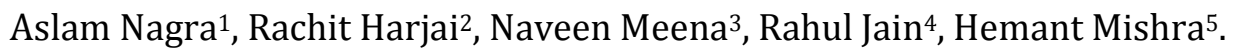

1. III Year PG Resident, Department of Radiodiagnosis \& Imaging, Mahatma Gandhi Medical College \& Hospital, Rajasthan.

2. Assistant Professor, Department of Radiodiagnosis \& Imaging, Mahatma Gandhi Medical College \& Hospital, Rajasthan.

3. III Year PG Resident, Department of Radiodiagnosis \& Imaging, Mahatma Gandhi Medical College \& Hospital, Rajasthan.

4. III Year PG Resident, Department of Radiodiagnosis \& Imaging, Mahatma Gandhi Medical College \& Hospital, Rajasthan.

5. Professor and Head of the Department, Department of Radiodiagnosis \& Imaging, Mahatma Gandhi Medical College \& Hospital, Rajasthan.

\section{CORRESPONDING AUTHOR}

Dr. Aslam Nagra

Department of Radiology,

Mahatma Gandhi Hospital,

Sitapura, Jaipur (302022), Rajasthan.

Email- aslamnagra@gmail.com

\section{HOW TO CITE THIS ARTICLE:}

Aslam Nagra, Rachit Harjai, Naveen Meena, Rahul Jain, Hemant Mishra "Persistent sciatic artery Aneurysm: A Rare Embryological vascular variant."Journal of Evolution of Medical and Dental Sciences 2013; Vol2, Issue 23, June 10; Page: 4166-4172.

ABSTRACT: Persistent sciatic artery (PSA) is a rare congenital vascular anomaly. The incidence of PSA is between 0.025-0.05 percent based on angiographic studies. The patient usually presents with the pulsatile swelling in the buttock or ischemic peripheral vascular disease. It can occur in two different forms either complete or incomplete, on the basis of the development of femoral artery and major vascular supply of lower limb. We report a case of a PSA with aneurysm and thrombus formation in a 60 year female with swelling in left gluteal region and lower extremity ischemic changes.

KEYWORDS: Persistent sciatic artery, Aneurysm, Ischemia.

INTRODUCTION: Persistent sciatic artery (PSA) is a rare congenital vascular anomaly ${ }^{1}$.The sciatic artery arises from the umbilical artery. During early embryonic period it supplies the blood to lower limb. The sciatic artery disappears at 12 to 14 weeks of intrauterine life and the superficial femoral artery takes over the vascular supply of the leg. Rarely, it may persist as the primary artery of the lower limb and continue into the popliteal artery. Persistence of the sciatic artery was first described by Green in the Lancet in 1832, on anatomic dissection 2. PSA is prone to atherosclerotic complications due to its anatomic location and repeated trauma at the site causing aneurysm or distal embolization ${ }^{3}$. 
CASE REPORT: A 60-year-old female came to our emergency department with painful, pulsatile swelling in left gluteal region since 2 years and darkening of great toe since 1 week. The patient was a tobacco chewer since 30 years. On physical examination, the swelling was seen in lower outer quadrant in left gluteal region and measured approx. 6 × $6 \mathrm{~cm}$ with ill defined margins. The swelling was non-tender, visibly and palpably pulsatile with no overlying skin changes. Left femoral \& popliteal arteries were weakly palpable and left dorsalis pedis artery was not palpable at all.Blood Pressure measured 170/110 mm Hg. Patient was anemic with hemoglobin of $9 \mathrm{gm} \%$ the rest of the serum and biochemical investigations were unremarkable.

Ultrasound of local swelling was done with a high frequency linear probe which revealed well defined, anechoic area of size $35 \times 33$ × $28 \mathrm{~mm}$ deep to a vessel (Figure 1). Continuous low velocity, monophasic flow was seen within the anechoic area and triphasic flow in the vessel above it on pulsed doppler. This excluded the initial clinical diagnosis of deep seated abscess.

Further evaluation was done with Computed Tomography-Angiography (CTA) which showed aneurysm formation of one of the branches of internal iliac artery with hypoplastic superficial femoral artery (Figure 2). The left tibial and dorsalis pedis artery were not visualized. The diagnosis on CTA was aneurysm of left Persistent Sciatic Artery with large crescent shaped thrombus and hypoplastic left superficial femoral artery.

DISCUSSION: Sciatic artery is a branch of umbilical artery and during early stages of embryonic development ( $6 \mathrm{~mm}$ stage) and is the principal vascular supply of the developing lower limb bud. The external iliac artery also arises from umbilical artery proximal to the origin of sciatic artery. During further development (12 mm embryo stage) external iliac artery divides into Common femoral and superficial femoral artery 4,5 . The superficial femoral artery descends up to the level of knee and then it terminates in a bifurcation into medial descending geniculate artery and superior communicating ramus branch ${ }^{5}$. The superior communicating branch joins the sciatic artery just above the knee. At this time the distal arteries are supplied by both sciatic and superficial femoral artery. Progressively as the embryonic development continues the flow through the femoral artery becomes prominent and the caliber of femoral artery becomes much greater as compared to sciatic artery. In the $3^{\text {rd }}$ month of intrauterine life (22-mm stage), the continuity of the sciatic artery is completely interrupted and the femoral artery alone carries blood to the branches beyond the $\mathrm{knee}^{5,6}$.

Several authors suggested that if the superficial femoral artery does not fully establish itself as the arterial inflow to the lower leg, the primitive sciatic artery may persist. However the cause of this incomplete development of superficial femoral artery is unknown. Anatomically course of PSA differs from that of superficial femoral artery. The PSA arises from internal iliac artery and runs through greater sciatic foramen below the piriformis muscle and lies adjacent to sciatic nerve in the gluteal region and thigh. In some cases it may lie within the sheath of sciatic nerve ${ }^{7}$. The sciatic nerve is therefore vulnerable to compression by sciatic artery aneurysm and may cause radicular pain. Prior evaluation with cross sectional imaging is must before planning any surgical or interventional procedure to see its relation with other anatomical structures.

Different classification of PSA is being proposed. Bower et $\mathrm{al}^{8}$ described persistent sciatic artery into complete and incomplete variety. In the complete type the sciatic artery is the main blood supply of the lower limb and superficial femoral artery is hypoplastic which ends in thigh. In incomplete type the superficial femoral artery is the main blood supply of the lower limb and it 
continues into popliteal artery whereas sciatic artery is hypoplastic and ends in thigh. Another classification was proposed by Pillet et al 9, 10 describing persistent sciatic artery into 4 categories (Table 1).

Patients with persistent sciatic arteries commonly present with a pulsatile gluteal mass which may be painful, especially in the sitting position. Aneurysm formation occurs in approximately $46 \%$ of cases 11 . The high incidence of aneurysm formation is probably related to repeated external trauma as the persistent sciatic artery is in a relatively vulnerable anatomic position. Other etiological factors include atherosclerosis, hypertension, and congenital lack of arterial elastic tissue. Other symptoms associated with a persistent sciatic artery aneurysm include sciatic neuropathy caused by sciatic nerve compression, and lower limb ischemia caused by thrombosis or distal embolization.

Conclusion: A persistent sciatic artery is a rare congenital vascular anomaly that can present with various atherosclerotic complications. Non-invasive procedures such as Doppler-US, CTangiography and MR-Angiography are important for correct diagnosis as well as to identify and determine the correct treatment options. Therefore, before undergoing any surgical or interventional procedure, a thorough and complete radiological evaluation of the peripheral arterial system is necessary. Multi-detector CT angiography helps in precise diagnosis and complete evaluation of complications caused by a persistent sciatic artery. It is the imaging modality of choice obviating the need of conventional angiography for the diagnosis. Also, the selective injection of contrast medium in the external iliac artery may cause an incorrect diagnosis of vessel occlusion due to the lack of enhancement of the popliteal artery (12). CT angiography can detect this rare embryological vascular variant non-invasively even in the presence of complete occlusion of the vessel.

TABLE 1: Pillet et al Classification of Persistent sciatic artery

\begin{tabular}{|l|l|}
\hline Type $\mathbf{1}$ & Complete Persistent Sciatic Artery with normally developed superficial femoral artery \\
\hline Type $\mathbf{2}$ & Complete Persistent Sciatic Artery with incomplete superficial femoral artery \\
\hline Type $\mathbf{3}$ & $\begin{array}{l}\text { Incomplete Persistent Sciatic Artery only upper part has persisted with normally } \\
\text { developed superficial femoral artery }\end{array}$ \\
\hline Type $\mathbf{4}$ & $\begin{array}{l}\text { Incomplete Persistent Sciatic Artery only lower part has persisted with normally } \\
\text { developed superficial femoral artery }\end{array}$ \\
\hline
\end{tabular}

\section{REFERENCES}

1) Van Hooft IM, Zeebregts CJ, van Sterkenburg SM, et al. The persistent sciatic artery. Eur J Vasc Endovasc Surg 2009; 37: 585-91 Epub 2009 Feb 20.

2) Green PH. On a new variety of the femoral artery. Lancet 1832; 1:730-2.

3) McLellan GL, Morettin LB. Persistent sciatic artery: clinical, surgical, and angiographic aspects. Arch Surg 1982; 117: 817-22. 
4) Papon X, Picquet J, Fournier HD, Enon B, Mercier P. Persistent sciatic artery: report of an original aneurysm-associated case. Surg Radiol Anat 1999; 21(2):151-3.

5) Senior HD. The development of the human femoral artery, a correction. Anat Rec 1920; $17: 271$.

6) Senior HD. The development of arteries of the human lower extremity. Am J Anat 1919; 25:55-95.

7) Arey LB. Developmental anatomy. 6th edn. Philadelphia: WB Saunders Co; 1954. p. 342 e59.

8) Bower EB, Smullens SN, Parke WW. Clinical aspect of persistent sciatic artery: report of two cases and review of the literature. Surgery 1977; 81(5):588-95.

9) Pillet J, Albaret P, Toulemonde JL, Cronier P, RaimbeauG,Chevalier JM. Troncarterielischiopoplite, persistancedel'artereaxiale. Bull Assoc Anat 1980; 64:109-22.

10) 12 Pillet J, Cronier P, Mercier Ph, Chevalier JM. The ischio popliteal arterial trunk: a report of two cases. AnatClin 1982; 3:329-31.

11) Ikezawa T, Naiki K, Moriura S, Ikeda S, Hirai M. Aneurysm of bilateral persistent sciatic arteries with ischemic complications: case report and review of the world literature. J VascSurg 1994; 20:96-103.

12) C. Michel, T. Richard, P. Jue Denis et al., "Aneurysm of the persistent sciatic artery," Journal de Radiologie, vol. 84, no. 12, pp. 1975-1977, 2003.

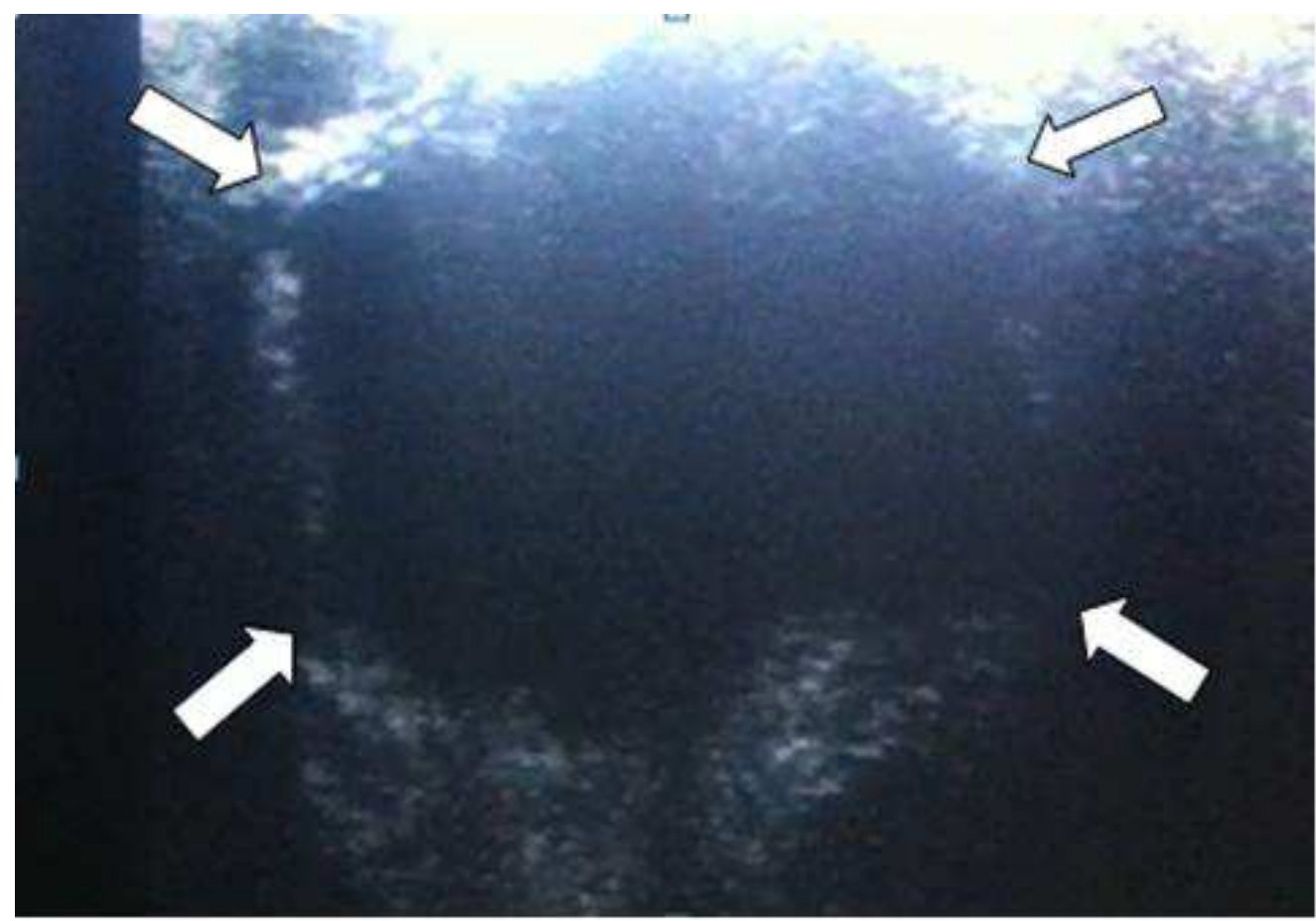

FIGURE 1: B-Mode Ultrasound of the local swelling reveals well defined anechoic area in left gluteal region. 


\section{CASE REPORT}

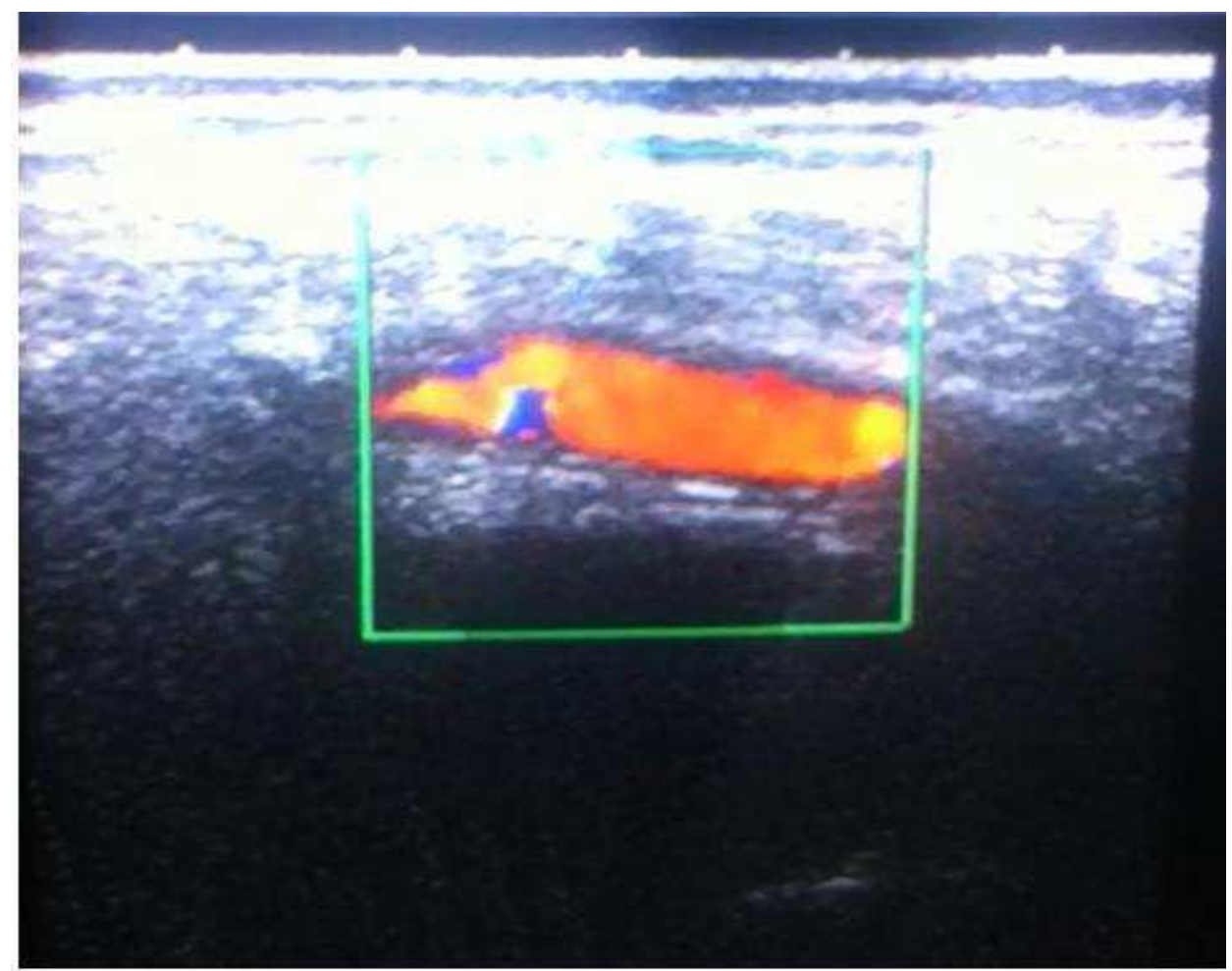

FIGURE 2: Color Doppler Ultrasound shows a vessel overlying the anechoic area in left gluteal region.

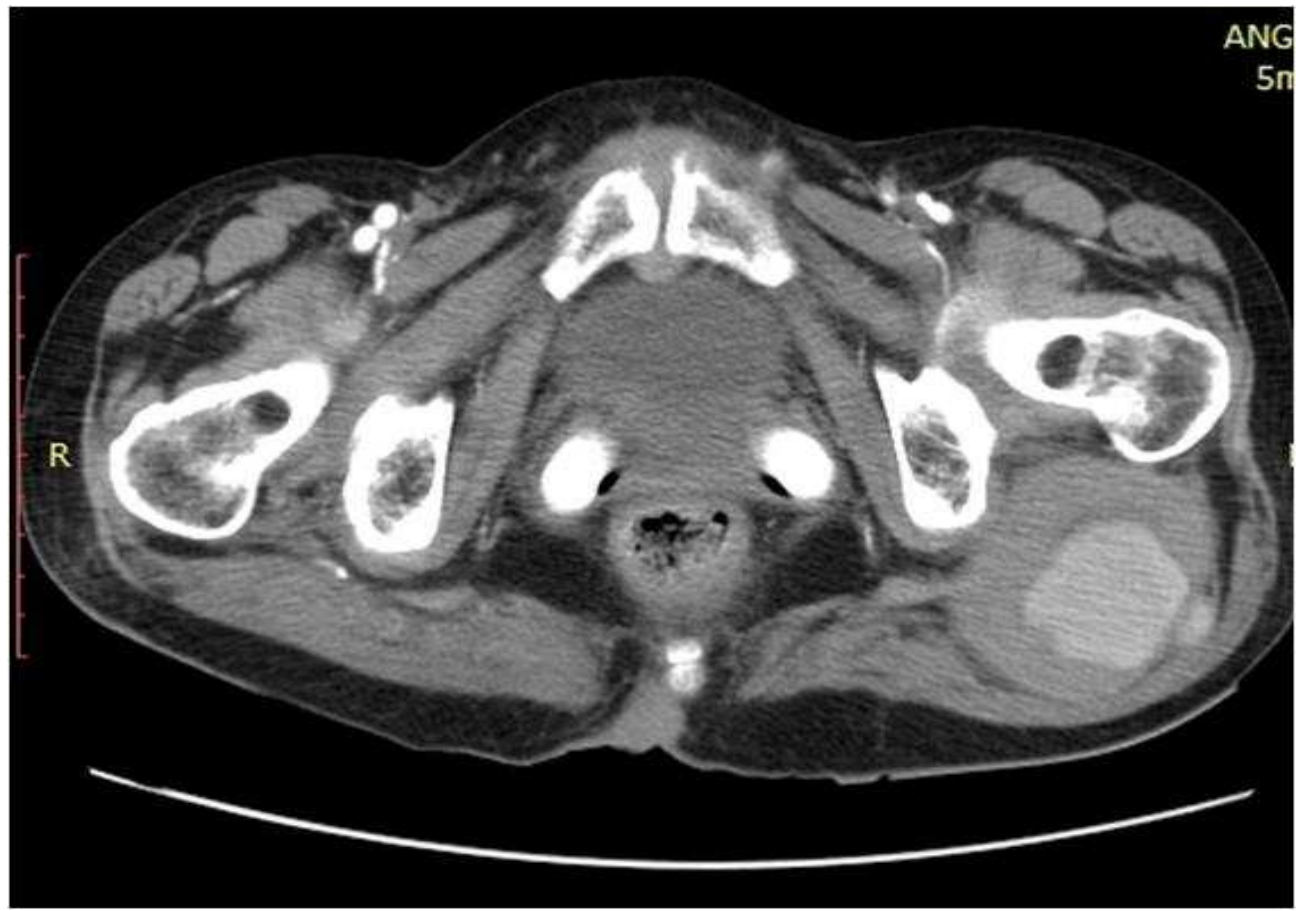

FIGURE 3: Axial Post-contrast CT image shows a well defined, rounded area filled up with IV contrast in left gluteal region with surrounding crescent shaped non-enhancing thrombus. 


\section{CASE REPORT}

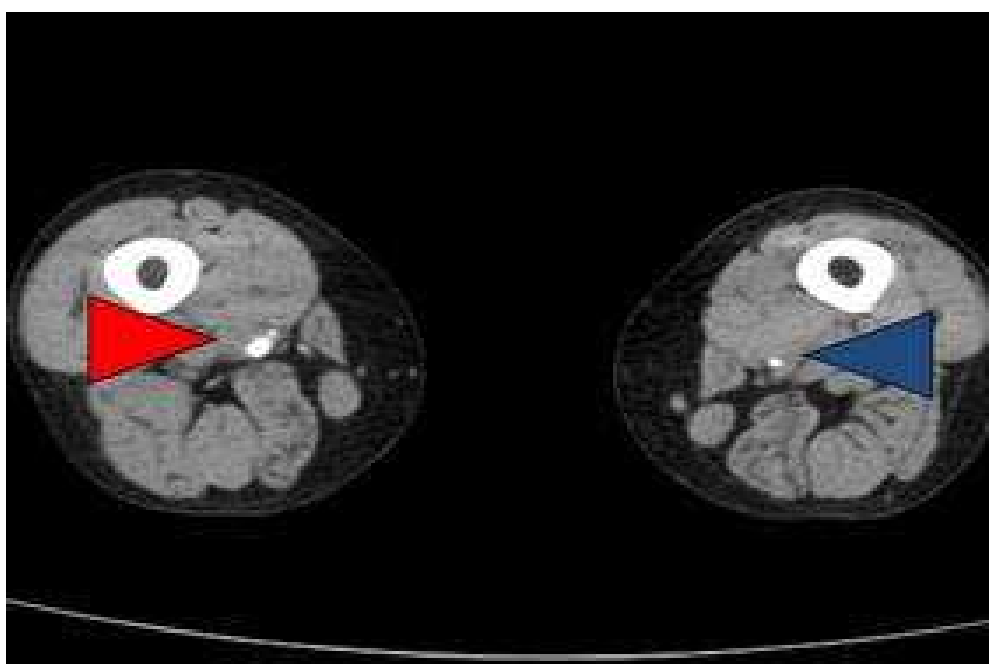

FIGURE 4: Axial Post-contrast CT image shows hypoplastic left superficial femoral artery.

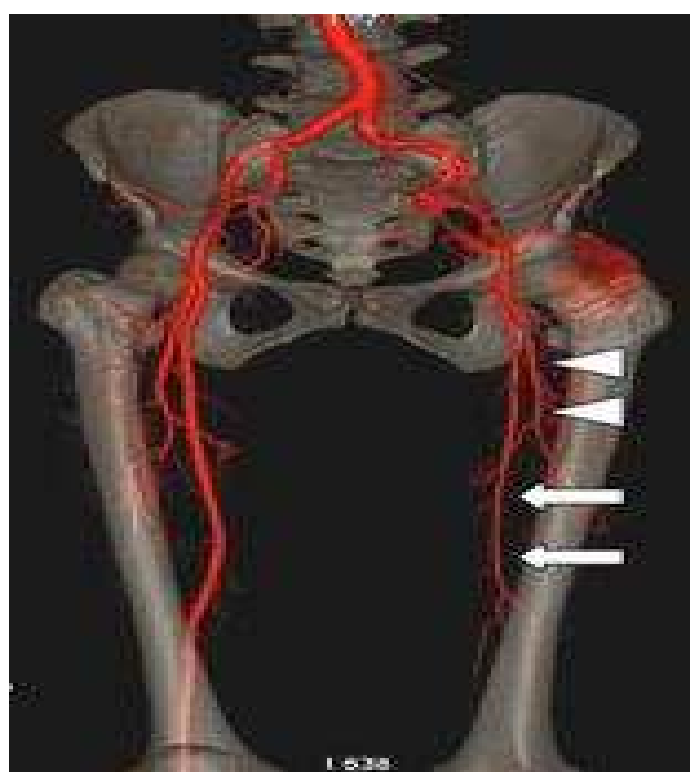

FIGURE 5: Volume Rendered CT Angiography image shows the hypoplastic left superficial femoral artery. 


\section{CASE REPORT}

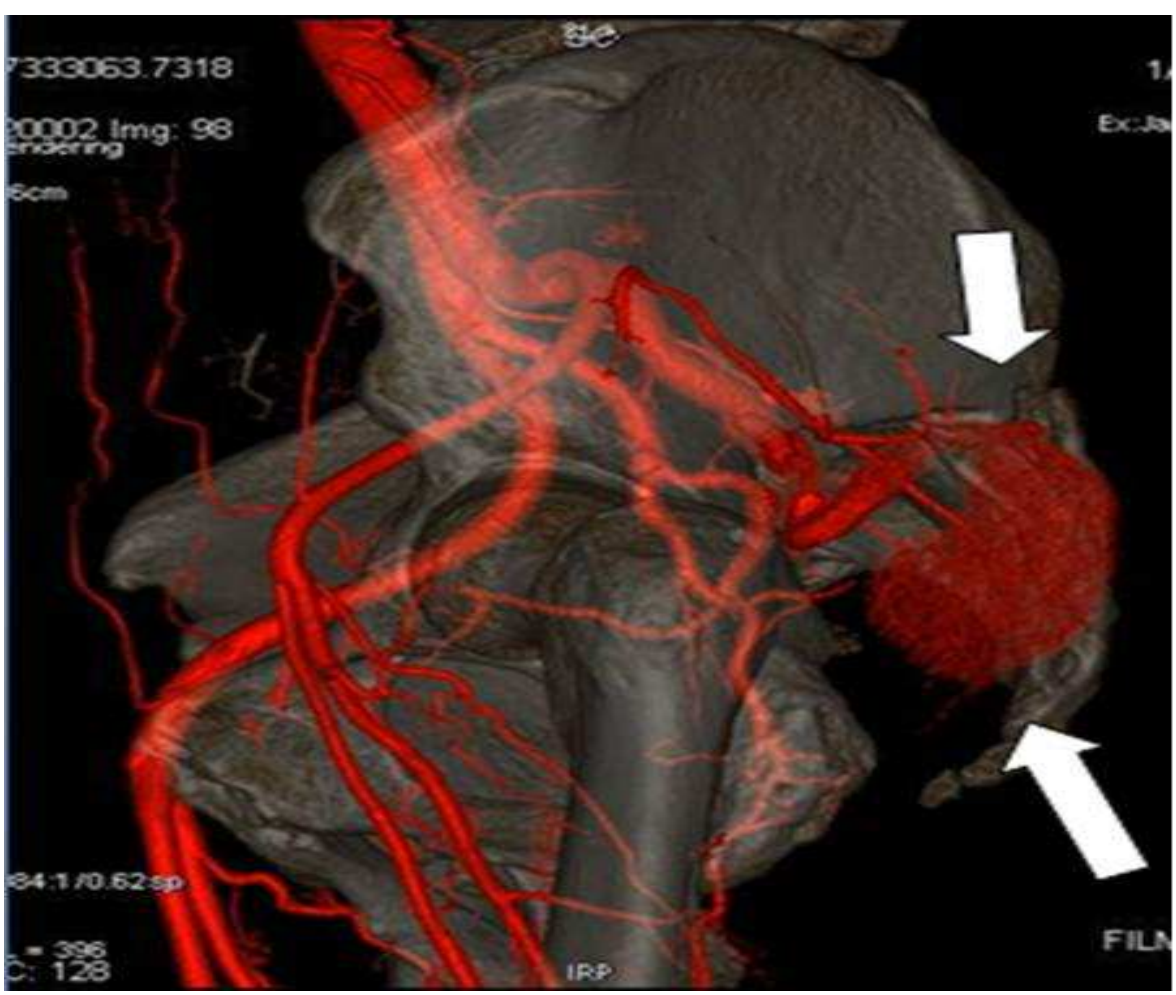

FIGURE 6: Volume Rendered CT Angiography image shows aneurysm formation in left gluteal region.

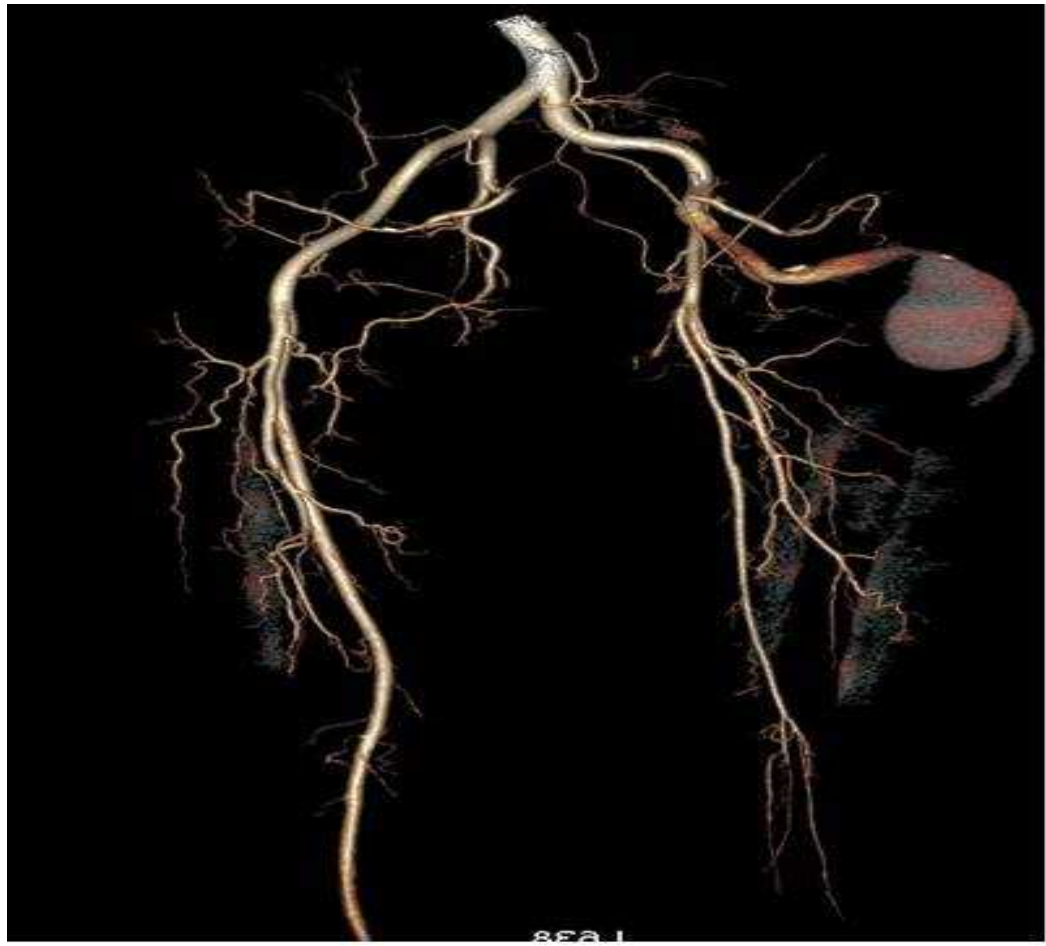

FIGURE 7: Volume rendered CT Angiography image after bone subtraction shows the aneurysm formation of persistent Sciatic artery and hypoplastic left superficial femoral artery. 